Journal for ImmunoTherapy of Cancer

\title{
Clinical outcomes in patients with metastatic renal cell carcinoma and brain metastasis treated with ipilimumab and nivolumab
}

\author{
Landon C. Brown (D , ${ }^{1}$ Kunal Desai, ${ }^{2}$ Wei Wei, ${ }^{3}$ Emily N. Kinsey, ${ }^{1}$ Chester Kao, ${ }^{1}$ \\ Daniel J. George, ${ }^{1}$ Brian I. Rini (D) , ${ }^{4}$ Moshe C. Ornstein (D) , ${ }^{5}$ Tian Zhang (D) ${ }^{1}$
}

To cite: Brown LC, Desai K, Wei W, et al. Clinical outcomes in patients with metastatic renal cell carcinoma and brain metastasis treated with ipilimumab and nivolumab. Journal for ImmunoTherapy of Cancer 2021;9:e003281. doi:10.1136/jitc-2021-003281

These data were presented at the 2020 ASCO Genitourinary Cancers symposium, Abstract \# 637.

Accepted 15 August 2021

\section{Check for updates}

(c) Author(s) (or their employer(s)) 2021. Re-use permitted under CC BY-NC. No commercial re-use. See rights and permissions. Published by BMJ.

${ }^{1}$ Department of Medicine, Center for Prostate \& Urologic Cancers, Duke Cancer Institute, Durham, North Carolina, USA

${ }^{2}$ Department of Internal Medicine, Cleveland Clinic, Cleveland, Ohio, USA

${ }^{3}$ Department of Quantitative Health Sciences, Cleveland Clinic Foundation, Cleveland, Ohio, USA

${ }^{4}$ Vanderbilt-Ingram Cancer Center, Nashville, Tennessee, USA

${ }^{5}$ Taussig Cancer Institute, Cleveland, Ohio, USA

Correspondence to

Dr Tian Zhang;

tian.zhang2@duke.edu

\section{ABSTRACT}

The combination of ipilimumab plus nivolumab $(\mathrm{I}+\mathrm{N})$ has greatly improved outcomes in patients with intermediate or poor-risk untreated metastatic renal cell carcinoma (mRCC). However, little is known about the outcomes of patients with brain metastasis (BrM) treated with $\mathrm{I}+\mathrm{N}$. A search was performed to retrospectively identify all patients with $\mathrm{mRCC}$ treated with $\mathrm{I}+\mathrm{N}$ in the Duke Cancer Institute and the Cleveland Clinic Taussig Cancer Center, followed by a chart review. Patients were included if they had BrM at the time of $\mathrm{I}+\mathrm{N}$ initiation. Cohort characteristics are summarized with descriptive statistics. Kaplan-Meier method was used to estimate overall survival (OS) and global, intracranial, and extracranial progression-free survival (PFS) for the cohort and log rank test was used to compare OS and PFS between patient groups. Radiographic response was categorized by RECIST. Fisher's exact test was used to correlate patient factors with radiographic response. From October 2017 to December 2020, 19 patients with BrM received I+N for mRCC with a median follow-up time of 27.1 months (range 15.0-35.6). By International Metastatic RCC Database Consortium (IMDC) risk criteria, 16\% had favorable, $58 \%$ had intermediate, and $26 \%$ had poor-risk disease. $68 \%$ were systemic therapy naïve, and $77 \%$ of patients had clear cell histology. 95\% had received local CNS directed therapy with surgery, radiotherapy, or both. The objective response rate was $44 \%$ ( $0 \%$ complete response) with three of six patients treated in the second line or greater setting experiencing a partial response. The median PFS was 7.6 months $(95 \% \mathrm{Cl} 5.6$ to 14.9$)$. The median extracranial PFS was 8.5 months $(95 \% \mathrm{Cl} 5.6$ to 19.7 ), and median intracranial PFS was 14.7 months (95\% Cl 7.2 to not reached). No variables assessed were significantly associated with radiographic response (gender, IMDC risk, presence of bone metastasis, line of therapy, or presence of immune related adverse events). In our retrospective cohort of patients with $\mathrm{MRCC}$ with $\mathrm{BrM}, \mathrm{I+N}$, in combination with CNS-directed local therapy, appears to have clinical efficacy as previously described with responses seen beyond the first-line setting. Further investigation is warranted in this population given exclusion from prior clinical trials.

\section{INTRODUCTION}

Metastatic renal cell carcinoma (RCG) will be responsible for an estimated 14830 deaths in the USA in 2020. ${ }^{1}$ The treatment landscape has changed dramatically with Food and Drug Administration approvals for multiple new front-line immune checkpoint inhibitor (ICI)-based combination therapies including ipilimumab plus nivolumab $(\mathrm{I}+\mathrm{N}),{ }^{2}$ pembrolizumab plus axitinib, ${ }^{3}$ avelumab plus axitinib, ${ }^{4}$ and nivolumab plus cabozantinib. ${ }^{5}$ Brain metastasis $(\mathrm{BrM})$ is a well-described complication of RCC with prior reports showing a 9.8\% cumulative 5-year incidence of $\mathrm{BrM}^{6}{ }^{6}$ No published data exist to support the efficacy of any of these ICI-based combination therapies in patients with $\mathrm{BrM}^{7}$ Checkmate 214, which investigated combination $\mathrm{I}+\mathrm{N}$ versus sunitinib, excluded all patients with central nervous system (CNS) metastasis while Keynote 426 (pembrolizumab/ axitinib), Javelin 101 (avelumab/axitinib), and Checkmate 9ER (nivolumab/cabozantinib) excluded patients with symptomatic or active CNS metastasis. Given the incidence and morbidity of $\mathrm{BrM}$, further investigation is needed to establish the optimal approach for patients with newly diagnosed metastatic RCC (mRCG) with BrM.

The only published clinical trial investigating patients with $\mathrm{BrM}$ in mRCC treated with ICI was GETUG-AFU 26 NIVOREN, ${ }^{8}$ which was a phase II single-arm study investigating the safety and efficacy of single agent nivolumab in patients who have progressed on prior antivascular endothelial growth factor targeted therapy. In total, on this study, 73 patients with $\mathrm{BrM}$ were identified, 39 of whom had target lesions in the brain and had not received local therapy with radiotherapy or surgery. The authors concluded that nivolumab activity was limited in patients 
with untreated $\mathrm{BrM}$ as the intracranial response rate was $12 \%$ among patients who had not received local therapy, emphasizing the importance of local therapy prior to systemic therapy. This contrasts to the experience in melanoma where combination ipilimumab and nivolumab has shown an objective response rate (ORR) to untreated CNS target lesions of 52\%. ${ }^{9}$ The efficacy of ipilimumab and nivolumab among patients with mRCC and BrM is unknown, and thus, we sought to perform a multicenter retrospective case series to review the outcomes and practice patterns among patients with mRCC and BrM treated with ipilimumab and nivolumab at two large academic medical centers.

\section{PATIENTS AND METHODS}

Patients with mRCC who were treated with combination ipilimumab and nivolumab from January 2016 to December 2020 were identified at Duke Cancer Institute (Duke) and Cleveland Clinic Taussig Cancer Center (CC). Patients were identified at Duke Cancer Institute performing a Duke Enterprise Data Unified Content Explorer search for all patients with RCC and treated with ipilimumab. Patients were identified at CC through a centralized database maintained by clinical providers. All patients identified underwent retrospective chart review and prespecified clinical variables were extracted with a data cut-off of December 31, 2020. This cohort was then narrowed by chart review to identify the cohort of patients with BrM. Patients were eligible if they had BrM known at the time of $\mathrm{I}+\mathrm{N}$ therapy. Notably, patients were included whether or not they had received prior intracranial local therapy (ie, surgery or radiotherapy) prior to $\mathrm{I}+\mathrm{N}$ administration.

Variables collected from the medical record include clinical features of the patients such as age, comorbidities, gender, and race. Disease-specific characteristics were collected such as stage at diagnosis, histology, and the presence of sarcomatoid features. Prior treatments including surgery, radiation, and prior systemic therapies were collected as well. Radiographic response was categorized per response evaluation criteria in solid tumors, RECIST (complete response (CR), partial response (PR), stable disease, progressive disease $(\mathrm{PD})$ ) by retrospective chart review performed by physicians based on radiology reports, imaging review, as well as primary clinician documentation. Progression was categorized as radiographic progression or unequivocal clinical progression as documented in the medical record. Progression-free survival (PFS) was defined as the time from initiation on $\mathrm{I}+\mathrm{N}$ to progression or death. Progression events were categorized as either extracranial or intracranial and separate extracranial and intracranial PFS was calculated. Overall survival (OS) was defined as the time from initiation on $\mathrm{I}+\mathrm{N}$ to death or censoring at last known time alive. Immune-related adverse events were documented, though grading was not performed.
Descriptive statistics were used to summarize patient characteristics and BrM-related variables. Fisher's exact test was used to correlate clinical factors with response status. Clinical variables assessed include gender, International Metastatic RCC Database Consortium (IMDC) risk group (favorable, intermediate, or poor risk), bone metastasis $(\mathrm{Y} / \mathrm{N})$, immune-related adverse event of any grade $(\mathrm{Y} / \mathrm{N})$, age (continuous), and number of prior therapies (continuous). Kaplan-Meier method was used to estimate OS and PFS, and log-rank test was used to compare OS and PFS between patient groups. Variables assessed for correlation with survival outcomes include age $(>v s<60$ years), gender, Eastern Cooperative Oncology Group performance status (ECOG PS, 0 vs $>0$ ), bone metastasis ( $\mathrm{Y}$ vs $\mathrm{N}$ ), lung metastasis ( $\mathrm{Y}$ vs $\mathrm{N}$ ), lymph node metastasis ( $\mathrm{Y}$ vs $\mathrm{N}$ ), infratentorial $\mathrm{BrM}$ ( $\mathrm{Y}$ vs $\mathrm{N})$, prior nephrectomy $(\mathrm{Y} v \mathrm{~N} \mathrm{~N})$, and number of prior therapies $(0 \mathrm{vs}>0)$. All tests were two sided and $p$ values of 0.05 or less were considered statistically significant. Statistical analysis was carried out using SAS Studio V.3.7 (SAS Institute) and R V.4.0 (R Foundation, Vienna, Austria).

\section{RESULTS}

A total of 19 patients were identified as having $\mathrm{BrM}$ due to $\mathrm{mRCC}$ and received I+N. Patients at Duke were identified using a query of the electronic medical records to search for patients with a diagnosis of RCC and having received ipilimumab which yielded 129 patients, which was narrowed to 122 who received $\mathrm{I}+\mathrm{N}$ for $\mathrm{mRCC}$. Of these 122 patients, 8 patients had BrM. At CC, 69 patients were identified as having received ipilimumab and nivolumab for mRCC by clinical care teams, of which 11 had BrM, for a final cohort of 19 patients.

Patient characteristics are summarized in table 1. Notable characteristics include a disproportionate overrepresentation of Caucasian $(n=17,89 \%)$ and male $(n=14$, $74 \%$ ) patients. Other characteristics include $77 \%$ clear cell histology, 58\% prior nephrectomy, and $84 \%$ stage IV at diagnosis. Zero patients were confirmed as having sarcomatoid histology. IMDC prognostic risk group breakdown included $26 \%$ poor risk, $58 \%$ intermediate risk, and $16 \%$ favorable risk. The majority of patients, $68 \%$ had received no prior systemic therapies for mRCC, while $11 \%$ had received one prior systemic therapy, and $21 \%$ had received two or more prior systemic therapies.

All patients had either BrM at the time of $\mathrm{I}+\mathrm{N}$ initiation or had BrM that were treated with local therapy just prior to treatment initiation. The majority of patients had received prior CNS directed local therapy with $4(21 \%)$ having received both surgery and radiotherapy to $\mathrm{BrM}$ and $14(74 \%)$ having received radiotherapy alone to BrM. Only one patient received no local therapy due to a high volume of extracranial disease. Radiotherapy was delivered as stereotactic radio surgery (SRS) in $17 / 18$ patients with one patient receiving whole brain irradiation. The vast of patients $(17 / 18)$ received local therapy before $\mathrm{I}+\mathrm{N}$ initiation with one patient receiving radiotherapy 
Table 1 Patient and treatment characteristics

\begin{tabular}{|c|c|c|c|c|c|}
\hline Baseline characteristics & $N(n=19)$ & $\%$ & Treatment characteristics & $N(n=19)$ & $\%$ \\
\hline Race & & & CNS-directed therapy & & \\
\hline Caucasian & 17 & 89.4 & None & 1 & 15.8 \\
\hline Other & 1 & 5.3 & Radiotherapy alone & 14 & 73.7 \\
\hline Gender & & & Other sites of metastasis & & \\
\hline Male & 14 & 73.7 & Liver & 2 & 10.5 \\
\hline Histology & & & Bone & 5 & 26.3 \\
\hline Clear cell & 15 & 79.9 & Lymph nodes & 14 & 73.7 \\
\hline Chromophobe & 1 & 5.3 & None & 1 & 5.3 \\
\hline Unclassified & 3 & 15.8 & No of brain metastasis & & \\
\hline No & 10 & 52.6 & $\geq 3$ & 6 & 31.6 \\
\hline Unknown & 9 & 47.4 & Brain metastasis location & & \\
\hline Prior radical nephrectomy & & & Supratentorial & 15 & 78.9 \\
\hline No & 8 & 42.1 & Infratentorial & 3 & 15.8 \\
\hline Yes & 11 & 57.9 & Both & 1 & 5.3 \\
\hline IMDC prognostic risk group & & & Ipilimumab doses $<4$ & 7 & 36.8 \\
\hline Poor risk & 5 & 26.3 & Received nivolumab maintenance & 12 & 63.2 \\
\hline Intermediate risk & 11 & 57.9 & Nivolumab doses, median (range) & $\begin{array}{l}6 \text { doses } \\
(1-21)\end{array}$ & \\
\hline$\geq 2$ & 4 & 21.1 & Immune-related adverse events & 12 & 63.2 \\
\hline Prior nivolumab monotherapy & 2 & 10.5 & & & \\
\hline
\end{tabular}

*ECOG PS, Eastern Cooperative Oncology Group performance status; CNS, central nervous system. IMDC, International Metastatic RCC Database Consortium.

shortly after initiation of cycle 1 of $\mathrm{I}+\mathrm{N}$. Concomitant sites of metastasis are noted in table 1 . The majority of patients had one metastatic lesion in the brain $(58 \%)$ with $42 \%$ having two or more lesions. Most patients were symptomatic from the BrM prior to treatment (68\%). All patients received at least one dose of ipilimumab, with $37 \%$ receiving fewer than four doses of ipilimumab either due to disease progression or toxicity.

Median follow-up time of patients alive in this cohort was 27.1 months (range 15-35.6) with the median time on therapy being 4.7 months (range 1-20.3). The median number of nivolumab doses, including those given concurrently with ipilimumab, was six doses (range 1-21). No patients were still receiving I+N or maintenance nivolumab with 18/19 (95\%) having experienced disease progression at last follow-up. Death had occurred in 7/19 patients $(37 \%)$. PFS and OS Kaplan-Meier curves are shown in figure 1. Median PFS was 7.6 months $(95 \%$ CI 5.6 to 14.9$)$, and the 12-month PFS rate was $26 \%$ (95\% CI $12 \%$ to $56 \%$ ). The median intracranial PFS was 14.7 months (95\% CI 7.2 to not reached, NR) with a 12-month intracranial PFS of $53 \%$ (95\% CI $34 \%$ to $81 \%$ ), and the median extracranial PFS was 8.5 months $(95 \%$ CI 5.6 to $19.7)$ with a 12-month extracranial PFS rate of $37 \%(95 \%$ CI $20 \%$ to $66 \%$ ). The median OS was not reached, with OS rates at 12 months and 24 months of $74 \%$ (95\% CI $56 \%$ to $96 \%$ ) and $68 \%$ (95\% CI $49 \%$ to $93 \%$ ), respectively. Clinically assessed best radiographic response rates are shown in table 2. All responses reported are extracranial responses due to the high rate of local CNS therapy 
A Overall Survival of All Patients, mRCC with Brain Mets

+ All

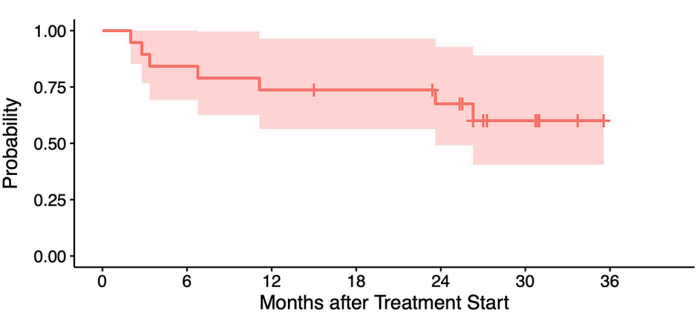

Number at risk
\begin{tabular}{|ccccccc}
19 & 16 & 14 & 13 & 11 & 5 & 0 \\
\hline 0 & 6 & $\begin{array}{l}12 \\
\text { Months after Treatment Start }\end{array}$ & 18 & 36
\end{tabular}

Cumulative number of events

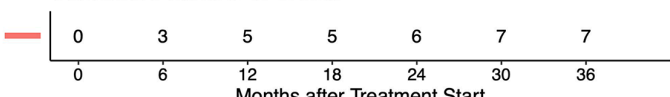

C Intracranial Progression-free Survival of All Patients, $\mathrm{mRCC}$ with Brain Mets

+ All

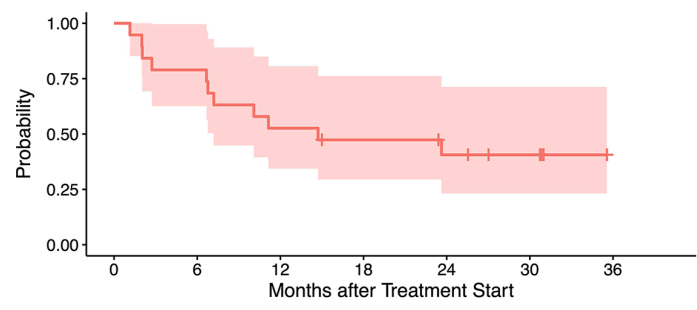

Number at risk
\begin{tabular}{|ccccccc}
19 & 15 & 10 & 8 & 6 & 4 & 0 \\
\hline 0 & 6 & 12 & 18 & 24 & 30 & 36
\end{tabular}

Cumulative number of events

\begin{tabular}{ccccccc}
0 & 4 & 9 & 10 & 11 & 11 & 11 \\
\hline 0 & 6 & 12 & 18 & 24 & 30 & 36 \\
& & & 11
\end{tabular}
B Progression-free Survival of All Patients,

mRCC with Brain Mets
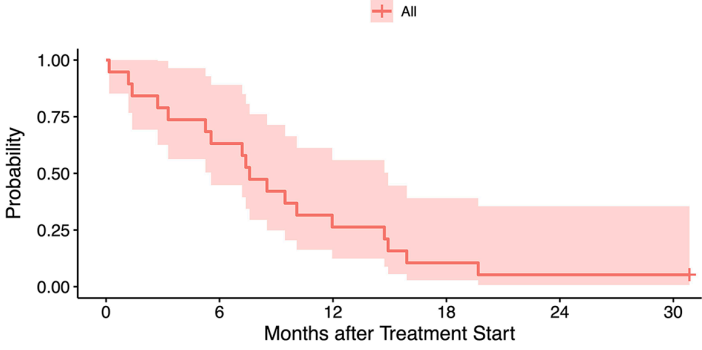

Number at risk
\begin{tabular}{|cccccc}
19 & 12 & 5 & 2 & 1 & 1 \\
\hline 0 & 6 & Months after Treatment Start & 24 & 30
\end{tabular}

Cumulative number of events

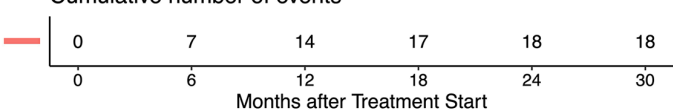

D Extracranial Progression-free Survival of All Patients, mRCC with Brain Mets

+ All

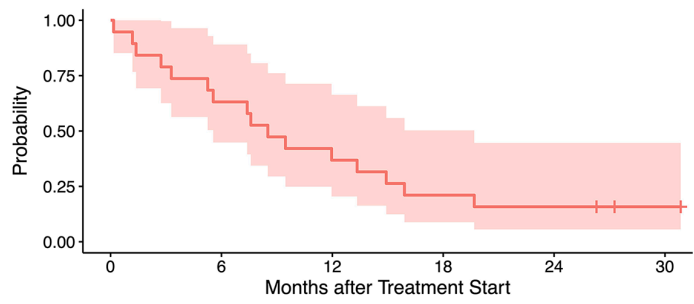

Number at risk

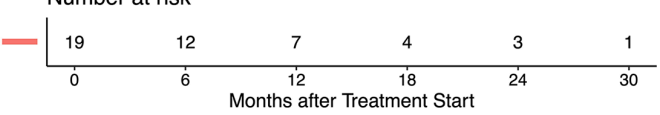

Cumulative number of events

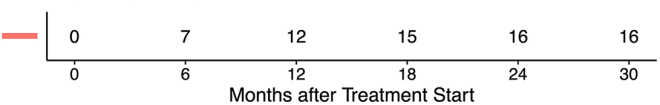

Figure 1 Kaplan-Meier analysis for (A) overall survival (OS), (B) progression-free survival (PFS), (C) intracranial PFS, and (D) extracranial PFS for patients with $\mathrm{mRCC}$ and brain metastasis treated with ipilimumab and nivolumab. mRCC, metastatic renal cell carcinoma.

administered prior to $\mathrm{I}+\mathrm{N}$ treatment. No CR was seen. A PR proportion of $44 \%(8 / 18)$ was seen with a PR proportion in the front line setting of $42 \%(5 / 12)$ and $50 \%$

Table 2 Best radiographic response for patients with $\mathrm{mRCC}$ and brain metastasis treated with ipilimumab and nivolumab among all patients, those treated in the first line setting and those treated in the second line or later setting

\begin{tabular}{llll}
\hline Variable & $\begin{array}{l}\text { All patients } \\
(\mathbf{n}=19)\end{array}$ & $\begin{array}{l}\text { First line } \\
(\mathbf{n}=13)\end{array}$ & $\begin{array}{l}\text { Second line or } \\
\text { later }(\mathbf{n}=6)\end{array}$ \\
\hline Best overall response, no. $(\%)$ & & \\
Complete response & 0 & 0 & 0 \\
Partial response & $8(42)$ & $5(38.5)$ & $3(50)$ \\
Stable disease & $6(32)$ & $5(38.5)$ & $1(17)$ \\
Progressive disease & $4(21)$ & $2(15)$ & $2(33)$ \\
Not evaluable & $1(5)$ & $1(8)$ & 0 \\
\hline
\end{tabular}

mRCC, metastatic renal cell carcinoma.
$(3 / 6)$ in the second line or later setting. The one patient who did not receive local CNS therapy had a large volume of extracranial disease and unfortunately died 2 months after initiation of therapy due to rapidly PD, without a response assessment of either intracranial or extracranial disease. Detailed toxicity data were not collected, though no CNS-related ICI toxicities were noted in this cohort. A swim-lane plot (figure 2) shows time on therapy as well as time to progression or death.

In this cohort, 12/19 patients did not experience intracranial progression. Of those 12 patients, 4 have died and 8 are still alive at last follow-up. Of the four who had died, the time from I+N initiation to death was $61,206,339$, and 719 days. Of the eight patients alive at data cut-off without intracranial progression, seven had experienced extracranial progression at a median follow-up of 878 days. CNS-directed radiotherapy alone had been administered in six of eight patients with two of eight having received both surgery and radiation for local therapy. 


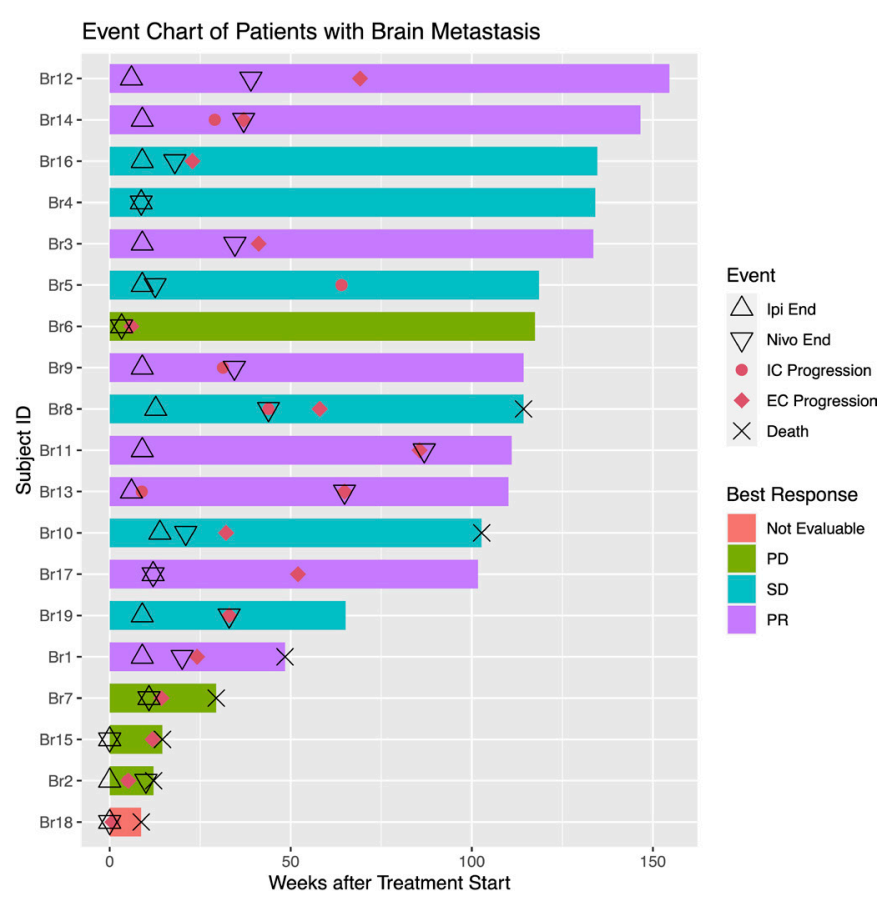

Figure 2 Swim-lane plot. PD, progressive disease; PR, partial response; SD, stable disease; IC, intracranial; EC, extracranial.

Seven patients had one single BrMs and one of eight patient had four sites of metastasis as compared with the overall cohort which had a mean of 1.7 (median 1, range 1-4) BrM sites. Other sites of metastatic disease among those without intracranial progression include seven of eight with lung metastasis, two of eight with bone metastasis, two of eight with liver metastasis, and one of eight with pancreas metastasis. IMDC risk categories were one of eight favorable, six of eight intermediate, and one of eight poor risk. All patients in this subgroup had clear cell histology and five of eight had prior nephrectomy.

An analysis to associate clinical variables with RR, PFS, and OS was performed (see methods for variables). No variables assessed were statistically associated with differential response rates by Fisher's exact method (data not shown). Having a prior nephrectomy $(\mathrm{n}=11)$ was associated with better OS compared with those with no prior nephrectomy $(\mathrm{n}=8)$ by the log-rank test $(\mathrm{p}=0.02)$ with 12 -month OS of $91 \%$ vs $50 \%$ and 24-month OS of $91 \%$ vs $33 \%$, respectively. PFS was numerically better but not statistically different by the log rank test among those with prior nephrectomy versus no prior nephrectomy (median PFS: 9.5 (95\% CI 7.6 to NR) vs 5.4 months (95\% CI 3.3 to NR), respectively). This numeric difference in PFS appeared to be primarily driven by the extracranial PFS with a median extracranial PFS of 13.3 months (95\% CI 8.5 to NR) among those with prior nephrectomy compared with 5.4 months (95\% CI 3.3 to NR) among those with no prior nephrectomy. The median intracranial PFS was similar between the two groups at 14.7 months (95\% CI 7.2 to NR) for those with prior nephrectomy vs 17.4 months (95\% CI 6.8 to NR) for those with no prior nephrectomy. Otherwise, no variables assessed were significantly associated with PFS or OS in this cohort.

\section{DISCUSSION}

Here, we report a multicenter experience of patients with $\mathrm{mRCC}$ and $\mathrm{BrM}$ treated with $\mathrm{I}+\mathrm{N}$. These data are consistent with the well-established practice of local CNS-directed therapy with either surgical resection of radiotherapy followed by systemic therapy, as $95 \%$ of patients in this cohort received CNS directed local therapy. The median extracranial PFS was shorter at 8.5 months compared with the median intracranial PFS at 14.7 months in this cohort of patients who have received aggressive local CNS therapy. Extracranial radiographic responses were seen with $\mathrm{I}+\mathrm{N}$, both in the front line and later line settings. Given the high rates of local therapy to BrM, we could not assess for intracranial radiographic responses to $\mathrm{I}+\mathrm{N}$. Though patients with $\mathrm{BrM}$ were excluded from the pivotal phase 3 Checkmate-214 clinical trial, these data suggest that local CNS-directed therapy followed by I+N is an acceptable strategy in the management of patients with mRCC metastatic to the brain.

This study adds to the limited literature describing outcomes among patients with mRCC and BrM. Recently, a cohort of patients with incidentally discovered asymptomatic BrM in the setting of mRCC at Gustave Roussy and Memorial Sloan Kettering Cancer Center was identified from patients being screened with mandatory brain imaging for clinical trial enrollment from 2001 to 2019. ${ }^{10}$ Of the 1689 patients screened, $72(4.3 \%)$ were noted to have BrM. Many patients were treated in the pre-ICI era and only $32 \%$ of patients were treatment naïve. These differences likely explain the worse survival outcomes observed in this cohort (12-month OS rate of 48\%) compared with our cohort (12-month OS rate of $74 \%$ ).

$\mathrm{BrM}$ is a grave manifestation of mRCC that can cause tremendous morbidity and requires a coordinated, multidisciplinary approach. For most metastatic solid tumors with $\mathrm{BrM}$, local therapy with either surgery, radiotherapy, or both followed by systemic therapy remains the cornerstone of treatment. Stereotactic radiosurgery has a more favorable side effect profile compared with traditional whole brain radiation. ${ }^{11}$ Advances in SRS technology have resulted in more patients being eligible for this approach, likely sparing many patients the toxicity of whole brain radiation therapy. The vast majority of patients in our cohort were not treated concurrently with radiation and ICI, but there is a growing body of literature to suggest that concurrent ICI and SRS is safe; there is also biological rationale for synergy between the two therapies. Two phase II clinical trials have been presented in abstract form describing prospective concurrent extracranial SRS and ICI therapy in mRCC. The NIVES trial treated 69 patients with mRCC with nivolumab single agent followed by extracranial SRS 7 days after the initial dose of nivolumab leading to an ORR of $17.4 \%$. Among these patients, $24.6 \%$ experienced grade $3-4$ toxicities, none 
within the radiation field. ${ }^{12}$ The RADVAX trial investigated the combination of extracranial SRS in 25 patients in between cycle 1 and 2 of combination ipilimumab and nivolumab and reported a $36 \%$ grade 3-4 toxicity rate and ORR of $56 \% .^{13}$ Additionally, retrospective data from patients with $\mathrm{BrM}$ in lung cancer have shown concurrent SRS with ICI therapy is well tolerated without increased toxicity. ${ }^{14}$ With regard to synergy between radiation and ICI, the abscopal effect is defined as tumor shrinkage outside of the radiation field after radiotherapy, theorized to be induced by radiation induced immunomodulation. This has been demonstrated in patients and mice with melanoma ${ }^{15}$ and anecdotally in RCC with BrM. ${ }^{16}$ Furthermore, Twyman-Saint Victor et al demonstrated that radiation may augment ICI by increasing the diversity of peripheral and intratumoral T-cell receptor clones, which can increase sensitivity to ICI. Further prospective randomized studies are needed before this approach is a standard of care, but the available data do suggest that concurrent SRS with ICI therapy is likely safe and may demonstrate synergy.

The strengths of these data are the 'real-world' nature of these treatments, though we recognize these patients were treated at two large, tertiary academic medical centers. These data have a number of limitations, primarily related to the retrospective nature of the investigation. We comprehensively assessed all patients treated with $\mathrm{I}+\mathrm{N}$ for mRCC at our institution, though we have no matched cohort of patients with $\mathrm{BrM}$ treated with alternative therapies for comparison. Clinical outcomes reported here are limited as they were assessed retrospectively based on clinical chart review and blinded RECIST evaluations are not available. Additionally, no genomic data were available for analysis from these patients and comprehensive genomic profiling was not routinely ordered on patients with mRCC at Duke or CC during this time period.

In conclusion, these data support the use of $\mathrm{I}+\mathrm{N}$ for patients with mRCC to the brain for systemic disease control following standard of care local therapies such as surgery and/or radiotherapy. Further prospective studies will be needed before considering omission of local CNSdirected therapy prior to systemic therapies or for concurrent local and systemic therapy administration.

\section{Twitter Brian I. Rini @brian_rini}

Acknowledgements The authors thank the patients and their families as well as the dedicated clinical and research staff at the Duke Cancer Institute and Cleveland Clinic Taussig Cancer Center.

Contributors LCB: writing-original draft, conceptualization, investigation. KD: investigation. WW: formal analysis. EK: investigation. CK: investigation. DG: writingreview and editing. BR: writing-review and editing. MCO: writing-review and editing. TZ: conceptualization, investigation, supervision, writing-review and editing.

Funding The authors have not declared a specific grant for this research from any funding agency in the public, commercial or not-for-profit sectors.

Competing interests LCB declares the following relationships: consulting with Seattle Genetics. KD declares the following relationship: consulting with Tyra BioSciences. WW declares that he has no conflicts of interest that might be relevant to the contents of this manuscript. EK declares that she has no conflicts of interest that might be relevant to the contents of this manuscript. CK declares that he has no conflicts of interest that might be relevant to the contents of this manuscript. DG declares the following relationships: Acerta Pharmaceuticals-Research, American Association for Cancer Research-Sr Editor, Astellas-Consultant, Research, Advisory Board, Astrazeneca - Consultant, Advisory Board, Axess OncologyIndependent Contractor, Bayer H/C Pharmaceuticals-Research, Consultant, Speaker, Honorarium, Travel accommodations, SC, BMS - Consultant, Research, Steering Committee, Calithera-Research, Capio Biosciences-Scientific Advisory Board, EMD Serono-Honorarium, Exelixis-Research, Consultant, Speaker, Honorarium, Travel accommodations, Flatiron-Consultant, Ipsen-Honorarium, Janssen Pharmaceuticals—-Research, Consultant, Independent Data Monitoring Committee (IDMC), Leidos Biomedical Research — Consultant, Merck Sharp \& Dohme - Consultant, Michael J Hennessey Associates-Honorarium, Consultant, Millennium Medical Publishing, Clinical Advances in Hematology \& Oncology-CoEditor-in-Chief, Modra Pharmaceuticals B.V.—Advisory Board, Myovant SciencesConsultant, Nektar Therapeutics-Steering Committee, Novartis-Research, Physician Education Resource - Consultant, Pfizer-Research, Consultant, Steering Committee, Honorarium, Sanofi-Research, Consultant, Speaker, Honorarium, Travel accommodations, UroGP0—Honorarium, UroToday-Honorarium, Travel accommodations, Vizuri Health Sciences-Consultant, NCl-Steering Committee. BR declares the following relationships: Research funding (to Vanderbilt University) from Pfizer, Hoffman-LaRoche, Incyte, AstraZeneca, Taris, Seattle Genetics, Arrowhead Pharmaceuticals, Immunomedics, BMS, Mirati Therapeutics, Merck, Surface Oncology, Dragonfly Therapeutics, Aravive and Exelixis; Consulting with Bristol Myer Squibb, Pfizer, Roche/Genentech, Aveo, Synthorx, Compugen, Merck, Corvus, Surface Oncology, 3DMedicines, Aravive, Alkermes, Arrowhead, GSK, Shionogi, Eisai. Stock ownership in PTC Therapeutics. MCO declares the following relationships: Consulting with BMS, Merck, Pfizer, Aveo, Exelixis and Eisai. TZ declares the following relationships: Research funding (to Duke University) from Pfizer, Janssen, Acerta, Abbvie, Novartis, Merrimack, OmniSeq, PGDx, Merck, Mirati, Astellas and Genentech; Speaking with Genomic Health and Sanofi Aventis; Consulting with AstraZeneca, Bayer, Pfizer, Foundation Medicine, Janssen, Merck, Amgen, MJH Associates, and BMS. Stock ownership/employment (spouse) from Capio Biosciences, Archimmune Therapeutics and Nanorobotics.

Patient consent for publication Not required.

Ethics approval This study was approved by the Duke University Health System IRB (Pro00101984) and Cleveland Clinic IRB (19-609/CASE2819).

Provenance and peer review Not commissioned; externally peer reviewed.

Data availability statement Data are available on reasonable request.

Open access This is an open access article distributed in accordance with the Creative Commons Attribution Non Commercial (CC BY-NC 4.0) license, which permits others to distribute, remix, adapt, build upon this work non-commercially, and license their derivative works on different terms, provided the original work is properly cited, appropriate credit is given, any changes made indicated, and the use is non-commercial. See http://creativecommons.org/licenses/by-nc/4.0/

\section{ORCID iDs}

Landon C. Brown http://orcid.org/0000-0002-5231-4859

Brian I. Rini http://orcid.org/0000-0002-2212-080X

Moshe C. Ornstein http://orcid.org/0000-0003-0184-3467

Tian Zhang http://orcid.org/0000-0001-8914-3531

\section{REFERENCES}

1 Siegel RL, Miller KD, Jemal A. Cancer statistics, 2020. CA Cancer J Clin 2020;70:7-30.

2 Motzer RJ, Tannir NM, McDermott DF, et al. Nivolumab plus ipilimumab versus sunitinib in advanced renal-cell carcinoma. $N$ Engl J Med 2018;378:1277-90.

3 Rini BI, Plimack ER, Stus V, et al. Pembrolizumab (pembro) plus axitinib (axi) versus sunitinib as first-line therapy for metastatic renal cell carcinoma (mRCC): outcomes in the combined IMDC intermediate/poor risk and sarcomatoid subgroups of the phase 3 KEYNOTE-426 study. J Clin Oncol 2019;37:4500.

4 Motzer RJ, Penkov K, Haanen J, et al. Avelumab plus axitinib versus sunitinib for advanced renal-cell carcinoma. $N$ Engl J Med 2019;380:1103-15

5 Choueiri TK, Powles T, Burotto M, et al. Nivolumab plus cabozantinib versus sunitinib for advanced renal-cell carcinoma. $N$ Engl $\mathrm{J}$ Med 2021;384:829-41.

6 Schouten LJ, Rutten J, Huveneers HAM, et al. Incidence of brain metastases in a cohort of patients with carcinoma of 
the breast, colon, kidney, and lung and melanoma. Cancer 2002;94:2698-705.

7 Kattan J, Rassy EE, Assi T, et al. A comprehensive review of the role of immune checkpoint inhibitors in brain metastasis of renal cell carcinoma origin. Crit Rev Oncol Hematol 2018;130:60-9.

8 Flippot R, Dalban C, Laguerre B, et al. Safety and efficacy of nivolumab in brain metastases from renal cell carcinoma: results of the GETUG-AFU 26 NIVOREN multicenter phase II study. J Clin Oncol 2019;37:2008-16.

9 Tawbi HA, Forsyth PA, Algazi A, et al. Combined nivolumab and ipilimumab in melanoma metastatic to the brain. N Engl $J$ Med 2018;379:722-30.

10 Kotecha RR, Flippot R, Nortman T, et al. Prognosis of incidental brain metastases in patients with advanced renal cell carcinoma. $J$ Natl Compr Canc Netw 2021:19:432-8.

11 Lamba N, Muskens IS, DiRisio AC, et al. Stereotactic radiosurgery versus whole-brain radiotherapy after intracranial metastasis resection: a systematic review and meta-analysis. Radiat Oncol $2017 ; 12: 106$
12 Masini C, lotti C, De Giorgi U, et al. Nivolumab (NIVO) in combination with stereotactic body radiotherapy (SBRT) in pretreated patients (PTS) with metastatic renal cell carcinoma (mRCC): first results of phase II NIVES study. J Clin Oncol 2020;38:613.

13 Hammers HJ, Vonmerveldt D, Ahn C, et al. Combination of dual immune checkpoint inhibition (ICl) with stereotactic radiation (SBRT) in metastatic renal cell carcinoma (mRCC) (RADVAX RCC). J Clin Oncol 2020;38:614.

14 Shepard MJ, Xu Z, Donahue J, et al. Stereotactic radiosurgery with and without checkpoint inhibition for patients with metastatic non-small cell lung cancer to the brain: a matched cohort study. $J$ Neurosurg 2019:1-8.

15 Twyman-Saint Victor C, Rech AJ, Maity A, et al. Radiation and dual checkpoint blockade activate non-redundant immune mechanisms in cancer. Nature 2015;520:373-7.

16 Levitin M, Ofori J, Shin WJ, et al. Radiation and checkpoint inhibitor immunotherapy lead to long term disease control in a metastatic RCC patient with brain metastases. Front Oncol 2020;10:566070. 